\title{
Scintillating Fibre and Radiation Damage Studies for the LHCb Upgrade
}

\section{Mirco DECKENHOFF ${ }^{* \dagger}$}

Technische Universitaet Dortmund (DE)

E-mail: mirco.deckenhoffetu-dortmund.de

\begin{abstract}
The Scintillating Fibre (SciFi) Tracker for the LHCb Upgrade is based on $2.5 \mathrm{~m}$ long multi-layered ribbons of $250 \mu \mathrm{m}$ diameter Kuraray SCSF-78MJ scintillating fibre as the active medium covering about $360 \mathrm{~m}^{2}$. The performance of the detector depends crucially on the geometrical and optical fibre parameters and, in particular, on their possible degradation due to ionising radiation. The dearth of results for this fibre type in the total ionising dose range of the upgrade up to $35 \mathrm{kGy}$ required a set of irradiation campaigns to estimate the behaviour of the full detector over its lifetime. This article presents results from studies of emission spectra, light yield, mirror technologies and the irradiation experiments performed by the LHCb SciFi collaboration over the last two years.
\end{abstract}

Technology and Instrumentation in Particle Physics 2014

2-6 June, 2014

Amsterdam, the Netherlands

\footnotetext{
* Speaker.

${ }^{\dagger}$ On behalf of the LHCb SciFi Tracker group.
} 


\section{Introduction}

A fast, high-resolution tracking system is needed for the upgrade of the LHCb detector. One component of its tracking system is a detector comprising 12 sensitive layers, each covering an area of about $5 \times 6 \mathrm{~m}^{2}$. This detector will be installed at the downstream side of the LHCb bending magnet. The sensitive layers will be mats which are made from $2.5 \mathrm{~m}$ long, $250 \mu \mathrm{m}$ diameter, round scintillating fibres. Details about the overall design and the components of this scintillating fibre (SciFi) tracker such as fibre read-out, electronics, cooling, mechanical support can be found in [1].

When a particle traverses the scintillating core of the fibre, it will excite the bulk material polystyrene (PS). The addition of two fluorescent dyes in the core improves the scintillation characteristics of the fibre. The primary dye increases the light yield and shortens the decay time of excited states in the PS. The secondary dye acts as a wavelength-shifter (WLS).

A small fraction of the scintillation light will be trapped in the fibre because of the lower refractive indices of the cladding materials compared to the fibre core. These photons will propagate to the fibre ends, see Figure 1. In the LHCb Upgrade SciFi Tracker, one fibre end will be read out by a silicon-photomultiplier (SiPM). A mirror will be attached to the opposite end of the fibre to increase the light yield at the SiPM.

The scintillating fibre SCSF78-MJ from Kuraray ${ }^{1}$ was chosen as baseline for the LHCb Upgrade SciFi tracker. Studies were carried out to reveal the emission spectrum of this fibre, the light yield, its radiation hardness and the benefit from the mirror.

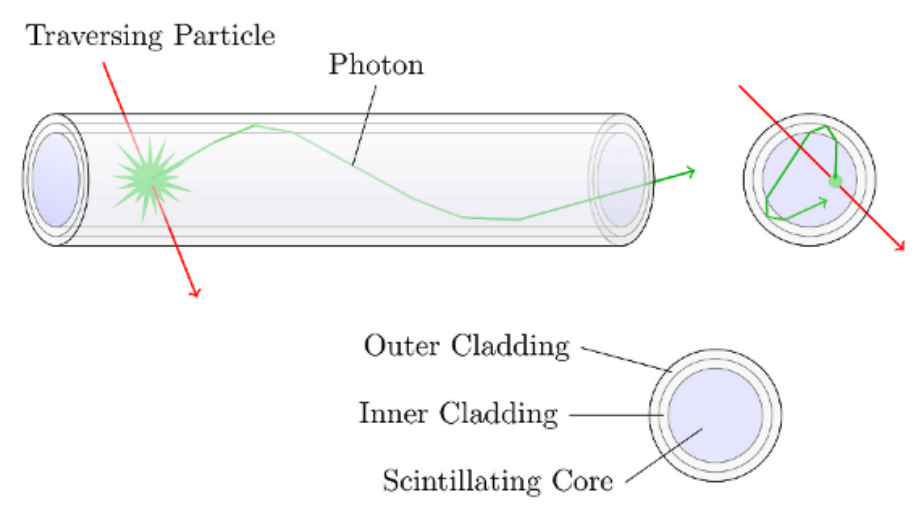

Figure 1: Lateral view and cross-section of a scintillating fibre with multiple claddings. The green line shows the path of an exemplary photon, which is produced by a traversing particle. The dimensions are not to scale. [2]

\section{Emission spectra}

Measured emission spectra of the fibre SCSF-78MJ are shown in Figure 2. The fibre was excited by the light of a UV LED at three different distances from the instrumented fibre end. The

\footnotetext{
${ }^{1}$ Kuraray Co., Ltd., Japan
} 
peak emission shifts slightly to longer wavelengths with increasing distance from the fibre end. Figure 2 illustrates also the wavelength-dependent photon detection efficiency (PDE) of KETEK ${ }^{2}$ and Hamamatsu ${ }^{3}$ SiPM. The measured emission spectra match the peak sensitivity of the sensors quite well.
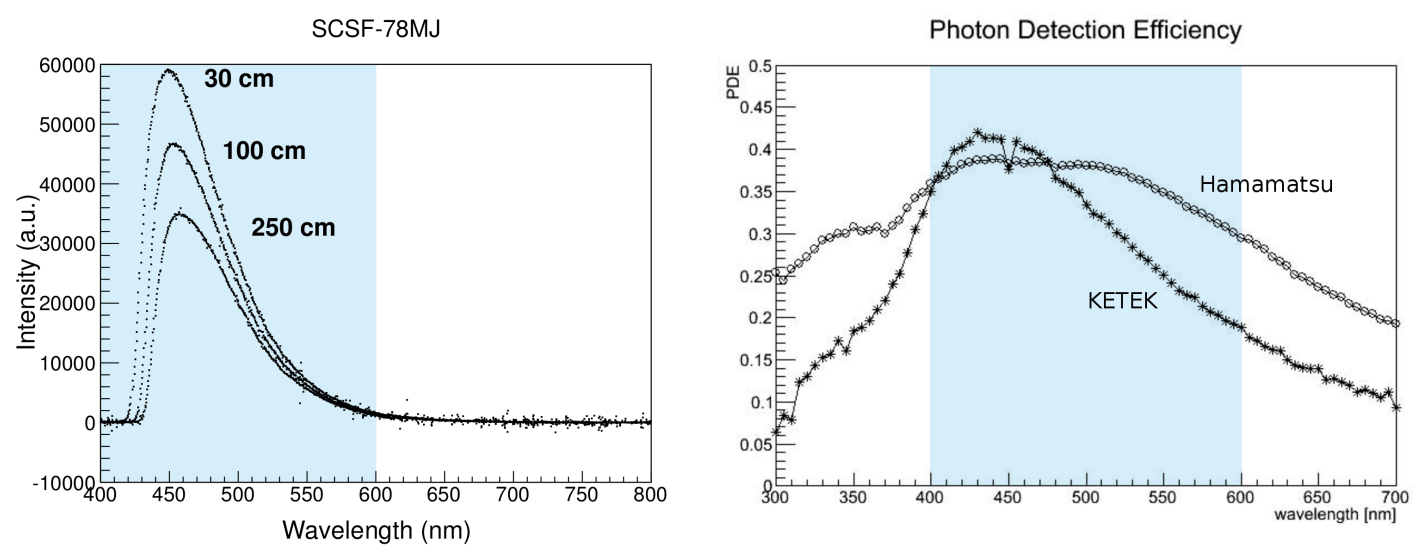

Figure 2: Left: Measured emission spectra of the SciFi SCSF-78MJ at three different distances from the fibre end. Right: Photon detection efficiency of Hamamatsu and KETEK SiPM. The shaded region shows the same wavelength interval from $400 \mathrm{~nm}$ to $600 \mathrm{~nm}$ in both plots. Details can be found in [1].

A Geant $4[3,4]$ simulation has been established to study the performance of the scintillating fibre [2]. It accounts for the emission spectrum of the primary dye, the WLS mechanism of the secondary dye and wavelength-dependent transmission losses. These fibre parameters and many more, e.g. the presence and reflectivity of a mirror at the non-instrumented fibre end, are read from a file at the start of the simulation program. Therefore their influence on the fibre performance can be studied without changing the source code and subsequent re-compilation. The simulated emission spectra at the fibre end, which are obtained by using literature data for the emission and absorption of the two dyes, show distributions similar to the measured data presented in Figure 2.

\section{Dye concentrations and light yield}

Only wavelength-shifted photons show a sufficiently low transmission loss in the SciFi. On the other hand, the secondary dye itself may contribute to the light attenuation due to self-absorption. However, Geant 4 simulations of a fibre with half and double concentration of the secondary dye with respect to the nominal concentration in the baseline fibre SCSF-78MJ show no significant impact on the attenuation lengths which are determined between $100 \mathrm{~cm}$ and $300 \mathrm{~cm}$ distant from the photo-detector, see Figure 3. Instead, the light yield increases with higher dye concentrations, since a higher fraction of scintillation photons will undergo the WLS process. Fibres with dye concentrations different from the nominal have been received from Kuraray recently. Measurements will be conducted to cross-check the simulation results.

\footnotetext{
${ }^{2}$ KETEK GmbH, Germany

${ }^{3}$ Hamamatsu Photonics K.K., Japan
} 


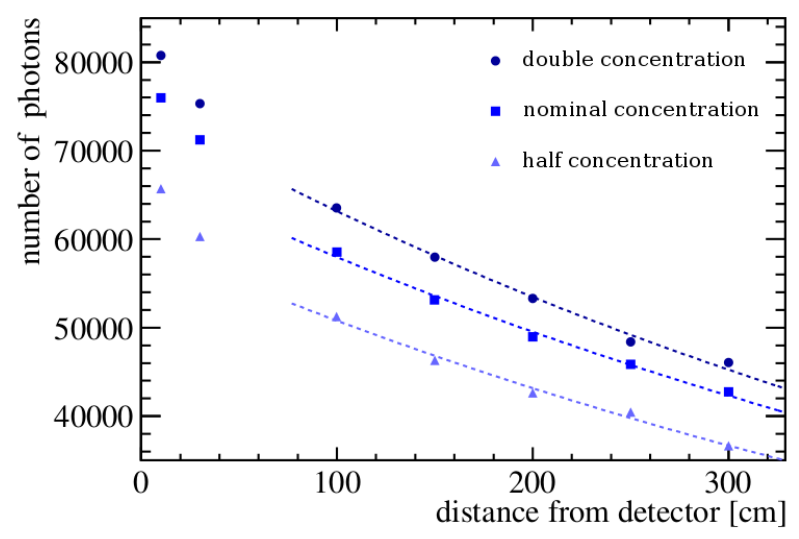

Figure 3: Simulated light output at one fibre end as a function of the distance of the excitation point from this end. The number of photons per $5 \mathrm{k}$ simulated minimum ionising particles is shown for three different concentrations of the secondary dye. [2]

The fibre mats used in the upgrade detector are proposed to be stacks of five fibre layers. A minimum ionising particle (MIP) crossing the fibre mat at the far end relative to the photodetector will have a light yield of about 15 to 20 photo-electrons. This number comprises the SiPM sensitivity and mirrored photons. The light yield is the same in measurements with test modules and simulation.

\section{Radiation damage}

The radiation environment in the $\mathrm{LHCb}$ cavern will degrade the fibres during the running period of the upgrade detector. The maximum accumulated dose expected from FLUKA simulations after the full runtime is $35 \mathrm{kGy}$ close to the beam pipe, which crosses the detector layers perpendicularly in their centres [5]. It decreases rapidly to values below $1 \mathrm{kGy}$ when moving $50 \mathrm{~cm}$ away from the beam pipe along the fibre axis.

The radiation-induced damage in polymer fibres is caused by complex processes of radical production and further reactions. These are dependent on many parameters, e.g. dose and dose rate, presence and diffusion of oxygen and time (due to possible annealing). An introductory overview of radiation damages in plastic scintillators can be found in [6]. Since there is a lack of information about radiation effects for SCSF-78MJ in literature, own studies were required.

Scintillating fibres were irradiated to various doses along their $3 \mathrm{~m}$ length by means of $24 \mathrm{GeV} / \mathrm{c}$ protons at CERN PS [7]. The measurement of light output before and after irradiation exhibits an attenuation length decreasing with dose. No significant differences between excitation with a UV LED and a Sr-90 source are observed. The UV LED is only capable of exciting the WLS dye, while the Sr-90 source initiates the whole scintillation process. This leads to the conclusion that no obvious decrease of scintillation light yield was induced by the irradiation.

Irradiation tests with $22.9 \mathrm{MeV}$ protons were performed at the Karlsruhe Institute of Technology (KIT) [8]. Two $\mathrm{m}$ long fibres were irradiated to four different doses along their axis. Constant 
doses were applied to $0.4 \mathrm{~m}$ fibres. The radiation-induced absorption ${ }^{4}$ shows a strong dependence on the photon wavelength. The damage per dose is consistent for different total doses within measurement uncertainties, see Figure 4. It follows that the damage increases linearly with dose under the test conditions.

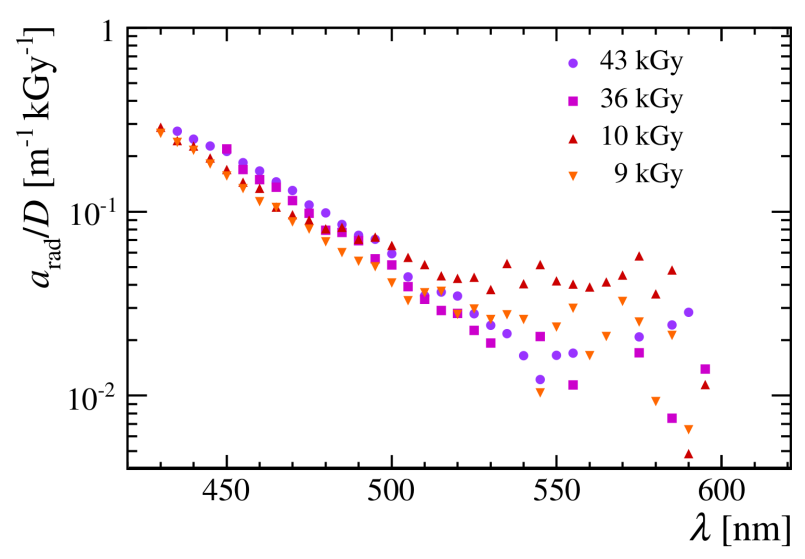

Figure 4: Additional radiation-induced absorption coefficient $a_{\text {rad }}$ per dose $D$ vs. photon wavelength $\lambda$ in scintillating fibre SCSF-78MJ after accumulation of different doses. The values are calculated from attenuation length data from [9]. The figure is taken from [2].

Three boards with $1.1 \mathrm{~m}$ long fibres were installed in the $\mathrm{LHCb}$ cavern close to the beam pipe for about half a year in 2012. These fibres received a non-uniform dose profile over their length at very low dose rates, which are comparable to the expected radiation in the upgraded detector.

Figure 5 shows a comparison of the results from the two proton tests and the in situ irradiation in the LHCb cavern. The following four empirical models are fitted to the CERN PS and KIT data points:

1. Damage linear with dose

$\Lambda^{\prime} / \Lambda_{0}=1 /(1+D / A)$

2. Power law

$\Lambda^{\prime} / \Lambda_{0}=1 /\left(1+(D / A)^{B}\right)$

3. Logarithmic

$\Lambda^{\prime} / \Lambda_{0}=\alpha+\beta \cdot \log (D)$

4. Exponential-like

$\Lambda^{\prime} / \Lambda_{0}=\exp \left(-(D / \alpha)^{\gamma}\right)$

In these equations $\Lambda^{\prime} / \Lambda_{0}$ denotes the ratio of attenuation lengths after to before irradiation, depending on the accumulated dose $D$. The variables $A, B, \alpha, \beta$ and $\gamma$ are free parameters of the fits. They are chosen to describe measured data empirically, i.e. they do not represent physical properties.

\footnotetext{
${ }^{4}$ The radiation-induced absorption coefficient $a_{\text {rad }}$ adds to the absorption coefficient $a$, which is observed in nonirradiated material. After irradiation the light intensity $I$ as a function of the photon path length $l$ can be described by $I(l)=I(0) \cdot \exp \left(-\left(a+a_{\mathrm{rad}}\right) l\right)$.
} 
However, all these models have limitations. They ignore the wavelength dependence of the absorption coefficient. Furthermore the measured attenuation length before irradiation $\Lambda_{0}$ varies between produced fibre batches and with distance from the photo-detector.

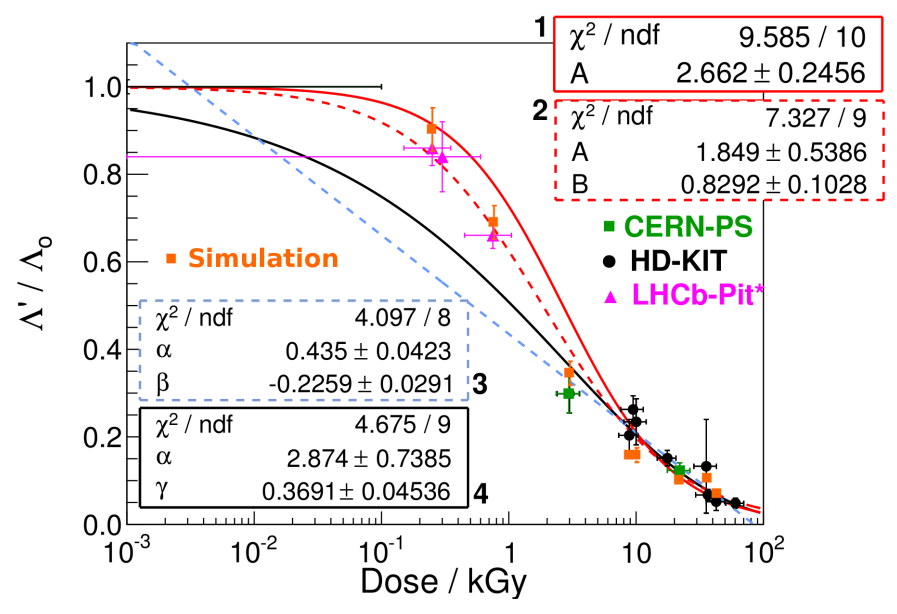

Figure 5: Ratio of attenuation length after to before irradiation $\Lambda^{\prime} / \Lambda_{0}$ versus dose $D$ [1]. Geant4 simulation data points are from [2]. LHCb pit data and simulated points are not included in the fits.

The excitation of irradiated fibres with dose profiles as they appeared in the mentioned tests was simulated in Geant4. This simulation accounts for a wavelength-dependent, radiation-induced absorption as shown in Figure 4. The resulting values of $\Lambda^{\prime} / \Lambda_{0}$ are in agreement with the measured data points.

At the current state of the radiation studies none of the four empirical models is considered to provide a better description of damages than the others. Each is consistent with the CERN PS and KIT data points, which are used in the fits.

Signal losses after the full runtime are estimated employing the exponential-like model 4 , since it is the most pessimistic at low doses. When the dose profile from FLUKA simulations at the innermost fibre is used and the effect of a mirror and integration time are considered, this leads to a signal loss of $27 \%$ for a maximum dose of $35 \mathrm{kGy}$ close to the beam pipe. Incorporating a safety factor of 2 due to possible uncertainties one gets a loss of $43 \%$ at $70 \mathrm{kGy}$ maximum dose.

\section{Mirror studies}

The use of a mirror at the end-face of the fibre opposite from the SiPM is essential to ensure a sufficient light output especially after irradiation. Therefore three mirror technologies were studied $^{5}$ :

${ }^{5}$ Details can be found in [10] 
- Aluminised Mylar ${ }^{6}$ (Al.M.) film, glue attached to the fibre ends

- 3M Enhanced Specular Reflector ${ }^{7}$ (ESR) film, glue attached to the fibre ends

- Thin film Aluminium (TFC) mirror, vacuum coated onto the fibre ends

The intensity ratio of the mirrored signal to the non-reflected light originating from an excitation at the mirror is shown in Figure 6. Two similar plates with scintillating fibres were used for this study. The reflectivity is obtained by a correction for Fresnel reflection which occurs even without use of a mirror. The best performance was observed with the two Aluminium mirrors. They show a reflectivity of about 0.85 .
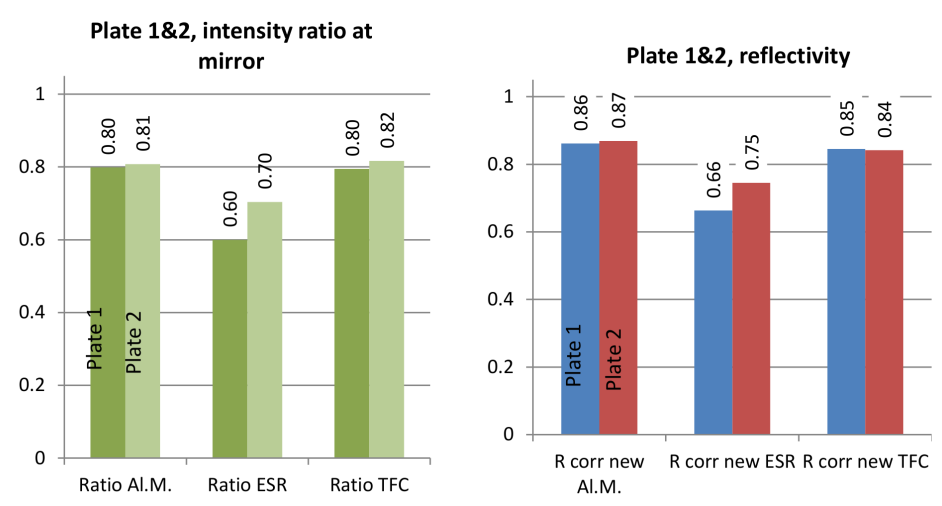

Figure 6: Intensity ratios of reflected to direct light and mirror reflectivity of the three technologies studied with two test plates [10].

The spectral reflectivity of these mirrors was determined with a commercial spectrometer of type Perkin Elmer Lambda 650. For geometrical reasons, the reflective foils and coatings were applied to scintillating plastic tiles instead of scintillating fibres.

\section{Conclusion}

A lot of R\&D has been done to examine the fibre performance in terms of light yield (including a mirror) and radiation hardness. So far, the baseline fibre SCSF-78MJ from Kuraray looks very suitable to build a large, high resolution tracking detector. There is still the need for more (precise) data for low dose and low dose rate irradiations, though. Tests with radioactive sources and X-ray are planned. Furthermore, the irradiation of a test module with the same support structure as in the upgrade detector is proposed. This aims for an irradiation in an environment which is closer to the upgrade scenario. There are possible improvements of the signal yield from higher dye concentrations. Measurements will be performed to cross-check the simulations.

\footnotetext{
${ }^{6}$ Mylar is a registered trademark of DuPont Teijin Films U.S. Limited Partnership

${ }^{7} 3 \mathrm{M}$ Company, USA
} 


\section{References}

[1] LHCb Collaboration, LHCb Tracker Upgrade Technical Design Report, CERN, Geneva, 2014, CERN-LHCC-2014-001, LHCB-TDR-015.

[2] M. Deckenhoff, PhD thesis, Technische Universitaet Dortmund, 2014, in preparation.

[3] Geant4 collaboration, S. Agnostelli et al., Geant4: a simulation toolkit, Nucl. Instrum. Meth. A506 (2003) 250 .

[4] Geant4 collaboration, J. Allison et al., Geant4 developments and applications, IEEE Trans. Nucl. Sci. 53 (2006) 270.

[5] N. Lopez March, M. Karacson, Radiation studies for the LHCb tracker upgrade, LHCb-PUB-2014-022, CERN-LHCb-PUB-2014-022, LHCb-INT-2013-003

[6] C. Zorn, A Pedestrian's Guide To Radiation Damage In Plastic Scintillators, Nuclear Physics B (Proc. Suppl.) 32 (1993) 377-383.

[7] C. Joram, T. Schneider, P. Shatalov, Measurements and radiation tests on scintillating fibres for the LHCb SciFi project, LHCb-PUB-2014-021, CERN-LHCb-PUB-2014-021, LHCb-INT-2013-002.

[8] B. Leverington, C. Joram, S. Baker, Scintillating Fibre Irradiation with 22.9 MeV Protons, LHCb-PUB-2014-024, CERN-LHCb-PUB-2014-024, LHCb-INT-2014-002.

[9] B. Leverington, private communication, 2013.

[10] C. Joram, T. Schneider, Mirroring of fibre ends for the LHCb SciFi project, LHCb-PUB-2014-020, CERN-LHCb-PUB-2014-020, LHCb-INT-2013-060. 